\title{
A Semi-Automated Approach for the Quantification of the Left Ventricle Chamber Volumes From Cine Magnetic Resonance Images
}

\author{
Claudio Fabbri ${ }^{1}$, Keigo Kawaji ${ }^{2}$, Noreen Nazir ${ }^{2}$, Amit Patel ${ }^{2}$, Victor Mor-Avi ${ }^{2}$, Cristiana Corsi ${ }^{1}$ \\ ${ }^{1}$ University of Bologna, Bologna, Italy \\ ${ }^{2}$ University of Chicago Hospitals, Chicago, IL (USA)
}

\begin{abstract}
Cardiac magnetic resonance imaging is the golden standard for the quantification of left ventricular function. However manual segmentation of the endocardium and epicardium boundaries has proven to be a time-consuming task, and the tools used in in clinical practice depend on the cardiologist's experience to assess the mitral valve plane, identifying papillary muscles and trabeculations. The goal of this work was to develop a new algorithm for the segmentation of the endocardium and epicardium of the left ventricle. To this end both a probabilistic and a Malladi-Sethian level-set models were used, followed by morphological operators and curvature flow to regularize the surfaces and exclude unconnected regions. The proposed algorithm and the ensuing workflow were applied to cine magnetic resonance data in 12 patients. Linear regression and Bland-Altman analysis for end-diastolic, end-systolic volumes and ejection fraction were performed, and clinical indexes derived from the surfaces were in good agreement with the ones obtained by manual tracings. This work provides the basis for faster and still accurate quantification of the cardiac function from cardiac magnetic resonance, and the basis for further processing aimed at the assessment of heart remodeling and diseases.
\end{abstract}

\section{Introduction}

Cardiovascular diseases are the leading cause of mortality in Western countries: The Global Burden of Disease estimates that $29.6 \%$ of all deaths worldwide (15616.1 thousands death) were caused by cardiovascular diseases in 2010 [1]. Among these stroke and coronary heart disease, in which arteries supplying blood to the heart muscle (myocardium) eventually leads to a heart attack (or myocardial infarction), is responsible for $12 \%$ of all deaths.

One of the most important technological developments of the last several decades which significantly contributed to early detection of heart disease is the use of noninvasive cardiac imaging, including a growing number of imaging modalities capable of depicting different aspects of cardiac anatomy and physiology.

Nowadays, Cardiac Magnetic Resonance (CMR) has already been proved to be and accurate tool for the quantification of the ventricular chamber volumes and the assessment of cardiac diseases [2]. By allowing the acquisition of the cardiac chambers with increasingly high in-plane spatial and temporal resolution, it minimizes problems such as geometric assumptions, foreshortening. However, it suffers of some issues associated with partial volumes, spacing between slices and metallic artefacts.

In clinical practice, all the available commercial tools require user input for the selection of anatomical landmarks, initialization or tracing of left ventricular (LV) boundaries. All these tasks are both quite time-consuming and rely on the user experience to include papillary muscles and other structures, with potential effect on the accuracy of calculated cardiac indexes. Therefore, the development of automated image analysis techniques that overcome these limitations and allow fast and fully automated, accurate quantification of these parameters without the need for operator intervention, is highly desirable.

As of today, a variety of methods have been proposed to achieve a full segmentation of the ventricular chamber in CMR [3], usually by analysing images slice by slice and frame by frame. Very few studies faced the issue of the most basal slice which is entirely included in the final surface for volume quantification, potentially resulting in volume overestimation.

To overcome the limitations of two-dimensional segmentation models, we designed and implemented a new approach based on a dynamic three-dimensional level-set formulation for automatic endocardium and epicardium surface detection. As a preliminary step, in this study, we validated the endocardium segmentation by comparing our estimates of end-diastolic, end-systolic volumes and ejection fraction against the ones derived by manual tracings of an expert radiologist. 


\section{Methods}

\subsection{CMR imaging and population}

Data from twelve patients (10 males, mean age: $63 \pm 10$ years) with heart failure, which already underwent CMR imaging for the assessment of heart function and scar burden were analyzed. Short-axis, 2-chamber, 4-chamber and 3-chamber ECG gated sequences were used; the sequences were acquired using a Philips 1.5 Tesla scanner.

The acquired image pixel spacings were ranging from 0.5 to $0.7 \mathrm{~mm}$; moreover, for the short-axis sequence, spacing between slices was $10 \mathrm{~mm}$ and slice thickness was $6 \mathrm{~mm}$. Temporal resolution was 30 frames per cardiac cycle.

Cine MR data were obtained from the Cardiac Imaging Laboratories at the University of Chicago Hospitals, these data were acquired using a Philips Scanner running at 1.5 Tesla. Reference measurements were obtained by manual tracing performed by an expert, using an in-house developed software package. LV trabeculations and papillary muscles were included within the left ventricle cavity, according to the American Society of Cardiologist guidelines [4].

\subsection{Image processing}

To constrain the segmentation inside the boundaries of the LV chambers a moving mitral valve (MV) plane was generated and the position of the apex was specified inside a three-dimensional rendering of 2-Chamber and 4Chamber views of the heart.

To generate the MV plane a set of at least three points was selected in the End-Diastole and End-Systole frames, every point in the ED is associated with another one in ES and then the position is interpolated during the cycle.

Then for every frame the plane that minimizes the distance between the set of points is calculated.

For the detection of the endocardial boundaries of the LV a probabilistic level-set was applied [5]. The model is initialized on all the planes between the MV plane and the apex, using and ellipsoid centered whose main axis is defined by the intersection of the 2-Chamber view plane and the 4-Chamber view. The differential equation that drives the level-set model considers the different resolution in the perpendicular direction (which is the spacing between slices) and inside the plane (given by the image pixel spacing).

Since the probabilistic model output not only detects the desired region of the LV but also other neighboring objects of similar intensity (such as the right ventricle), a series of morphological operator and connectivity checks were applied to the segmentation.

First every unconnected region was erased, then the
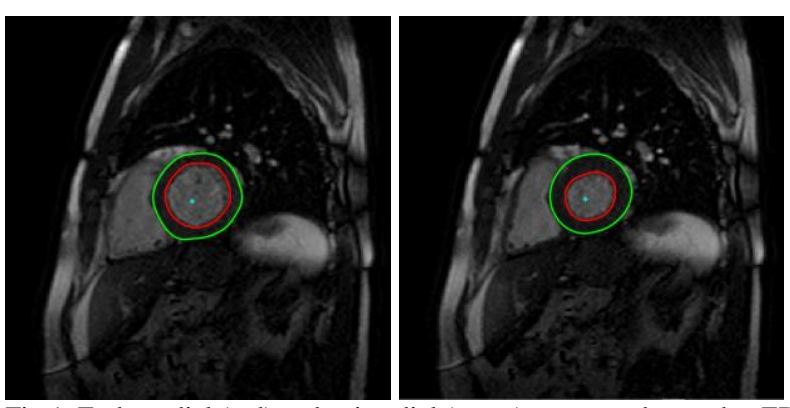

Fig 1. Endocardial (red) and epicardial (green) contours detected at ED (left) and ES (right) on slice at the mid level of the left ventricle.

center and size of the segmented region were calculated in each plane and if the difference between following frames was too big the segmentation was adjusted using the mask obtained from the previous frame, then a regularization step using curvature flow was applied to smooth the resulting boundaries.

The resulting segmentation is then used to initialize the level-set model in the following frame, cutting the time needed to reach the end of the level-set evolution.

After the segmentation of the endocardium boundaries is completed, a Malladi-Sethian level-set model [6] is applied to detect the epicardium. The level-set function used as initial condition for the model is obtained by reinitializing the previously obtained contours and adding an offset to ensure that the solution adheres to the epicardium boundaries.

Statistical analysis was performed using Excel, Correlation Analysis was conducted for the End-Diastolic Volume, End Systolic Volume and the Ejection Fraction.

\section{Results}

The tool for dynamic volume and mass quantification from was developed and implemented in MATLAB and tested on a laptop with an i7-7600M Processor with 16 GB of Ram.

Time required for the segmentation of a full Cine sequence depends on its size and on the number of frames acquired. Average time for a data set of 40 frames, 10 slices and 200 voxel side was about five minutes.

End-diastolic and end-systolic volumes measured from conventional semi-automated tracings ranged widely from 78 to $176 \mathrm{ml}$ and from 48 to $124 \mathrm{ml}$, respectively, reflecting the inhomogeneity of the study group.

An example of detected endocardial and epicardial contours at mid-level of the ventricle is shown in Figure 1.

In Figure 2 there are the resulting graphs with linear regression and Bland-Altman analysis between the proposed algorithm and a manually traced reference for EDV and ESV. Linear regression analysis between the proposed approach and the reference volume values 

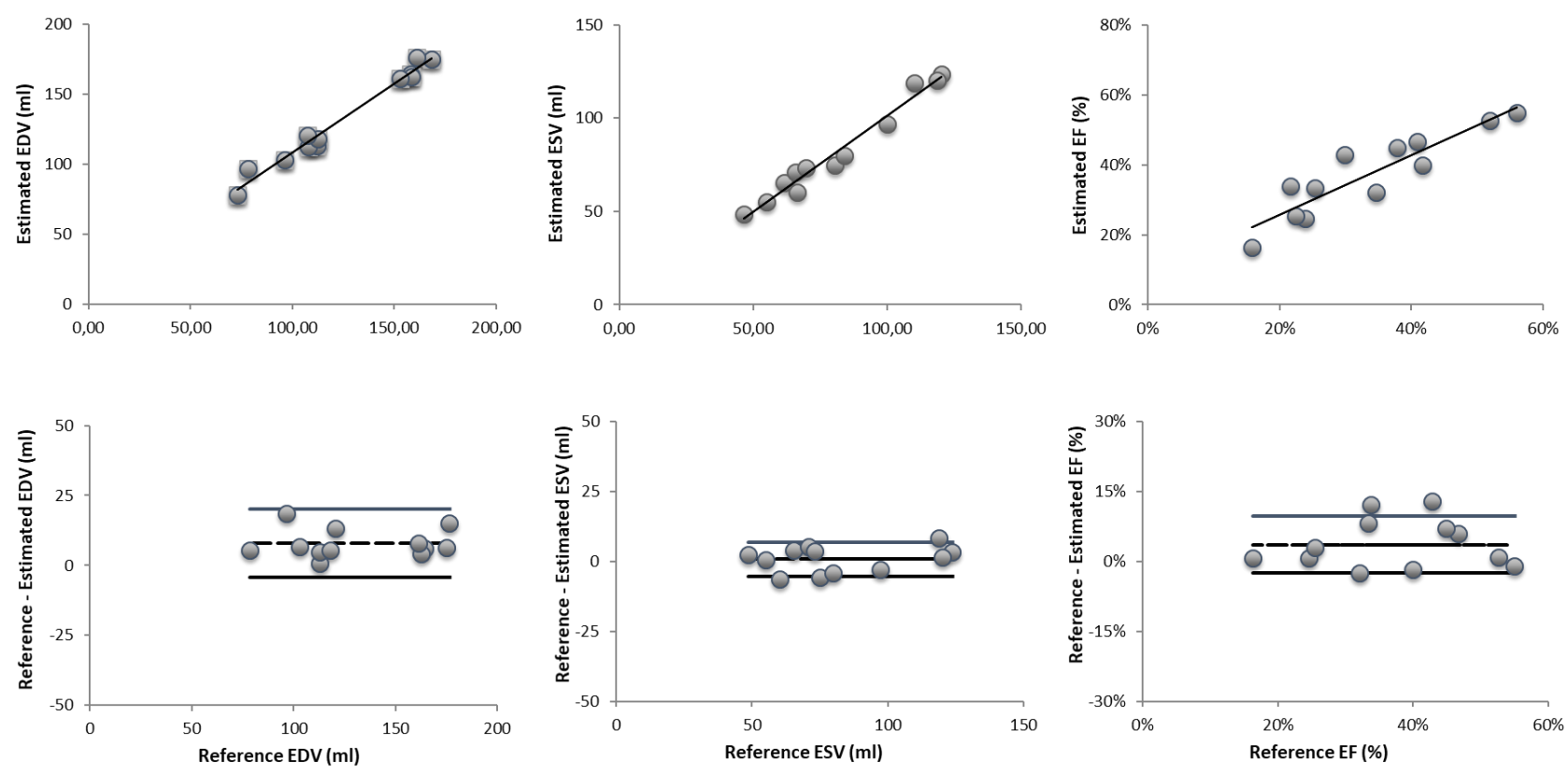

Fig 2. Correlation analysis (top row) and Bland-Altman plots (bottom plots) between EDV, ESV, EF computed applying the proposed approach and reference values from manual tracings.

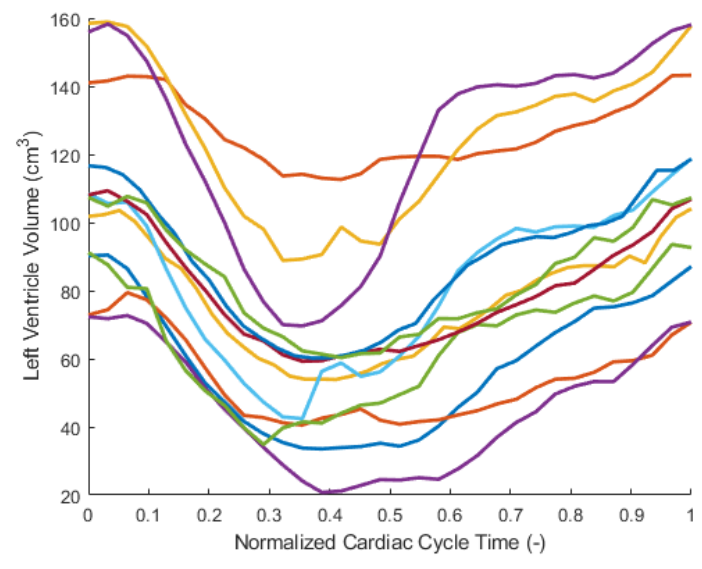

Fig 3. Volume-Time curves for the twelve patients analyzed in this study

resulted in very good correlation coefficients and regression slopes near 1.0 for both end-diastolic and endsystolic volumes (EDV: $\mathrm{r}=0.99, \mathrm{y}=0.9 \mathrm{x}+15.3$; $\mathrm{ESV}$ : $\mathrm{r}=0.94, \mathrm{y}=1.0 . \mathrm{x}+2.7)$. LV ejection fraction resulted in a correlation coefficient of $\mathrm{r}=0.91, \mathrm{y}=0.85 \mathrm{x}+0.1$ ). BlandAltman analysis shows small biases between the computed parameters and the reference technique for EDV, ESV and EF (bias: $7.9 \mathrm{ml} ; 0.8 \mathrm{ml}$ and $4 \%$ respectively). These biases reflected systematic errors of $6.0 \%, 1.0 \%$ and $10.3 \%$ of the corresponding mean values. The 95\% limits of agreement were relatively narrow (EDV: $9.7 \mathrm{ml}$, ESV: $8.8 \mathrm{ml}$, EF: 10\%), corresponding to
Volume time curves are available through the cardiac cycle as shown in in Figure 3.

\section{Discussion and conclusion}

The proposed algorithm allows a faster quantification of the LV chamber volume throughout the cardiac cycle compared to the time-consuming manual measurements limited to end-diastolic and end systolic frames for cardiac function evaluation. Importantly the proposed approach makes available volume-time curves thus allowing diastolic and systolic function in addition to ejection fraction.

Moreover, the proposed approach provides a solution for partial volume error correction, through the definition of a moving MV plane which eliminates the need for subjective slice selection and ensuing errors in LV volume measurements.

Validation of the new designed approach against volumes derived from manual tracings from expert cardiologist's shows promising results for EDV. ESV quantification showed lower levels of agreement probably as a consequence of a partial volume effect affecting reference ESV values, in which the basal slices are entirely considered. Consequently, also EF suffers from the same potential issue.

In the next future, the three-dimensional models will be integrated with data from other sequences already available, such as delayed enhancement or fast strain 
encoding ones to allow further analysis of the cardiac tissue and overall heart function.

\section{References}

[1] OECD, Health at a Glance 2013: OECD indicators, Technical report, 2013.

[2] C. Petitjean and J.-N. Dacher, "A review of segmentation methods in short axis cardiac MR images.," Medical image analysis, vol. 15, no. 2, pp. 169-84, Apr. 2011.

[3] D. Wei, Y. Sun, S.-H. Ong, P. Chai, L. L. Teo, and A. F. Low, "Three-dimensional segmentation of the left ventricle in late gadolinium enhanced MR images of chronic infarction combining long- and short-axis information.," Medical image analysis, vol. 17, no. 6, pp. 685-97, Aug. 2013.

[4] M. D. Cerqueira, "Standardized myocardial segmentation and nomenclature for tomographic imaging of the heart: A statement for healthcare professionals from the Cardiac Imaging Committee of the Council on Clinical Cardiology of the American Heart
Association," Circulation, vol. 105, no. 4, pp. 539-542, Jan. 2002.

[5] A. Sarti, C. Corsi, E. Mazzini, and C. Lamberti. "Maximum likelihood Segmentation of ultrasound images with Rayleigh distribution", IEEE Transactions on ultrasonics ferroelectrics and frequency control, vol. 52, no.6, pp. 947-960, 2005.

[6] R. Malladi, J. A. Sethian, B. and C. Vemuri, "Shape modeling with front propagation: A level set approach", IEEE Trans pattern analysis and machine intelligence, vol. 17, no. 1, pp. 158-175, 1995.

Address for correspondence

Claudio Fabbri

University of Bologna

Viale Risorgimento 2, 40136 Bologna, Italy

E-mail: claudio.fabbri10@unibo.it 